\title{
Transient Electromagnetic Torque Calculation Method for Short Circuit Fault of Turbo Generator Set
}

\author{
Haihua Zhang ${ }^{1, \text { a }}$, Shiwu Xiao ${ }^{1, b}$,Xiaoqing Xiao $^{2, c}$ and Likun Zheng ${ }^{2, d}$ \\ ${ }^{1}$ North China Electric Power University, Beijing \\ ${ }^{2}$ Guangdong Electric Power Research Institute, Guangzhou \\ azhhcarl@163.com, bxiaoshiwu@263.net, '986426823@qq.com, d909394537@qq.com
}

\begin{abstract}
Keywords: Sub synchronous oscillation. Transient electromagnetic torque. IEEE first standard model.

Abstract. This paper presents a mathematical model of the fault component network for systems with series compensation devices, when the three-phase short circuit occurs. After the transient electromagnetic torque is calculated, the initial amplitude and attenuation coefficient of transient electromagnetic torque are derived. Based on the first standard model of IEEE, Simulation analysis have been done under the situation of different fault locations and whether the fault circuit contains series compensated capacitors. Simulation results are consistent with previous calculation results.
\end{abstract}

\section{Introduction}

Transient torque amplification problem caused by sub synchronous oscillation should take into account the interaction between the electromagnetic characteristics of the AC transmission system and the mechanical characteristics of the generator shaft. Large disturbances are also strongly nonlinear and cannot be linearized. It is very difficult to research the transient torque amplification.

Reference [1] gives the definition of transient torque amplification, references [2,3] use Fourier transform, Prony Analysis, Hilbert - Huang transform method, wavelet analysis and damped sine atomic decomposition to identificate sub-synchronous oscillation modes, but none of these references analyze the physical nature of transient torque amplification.

References [4,5] use time domain simulation method to study the transient torque amplification, and the curve of variables change over time is obtained, but it cannot be analyzed on sub synchronous oscillation on principle, and the analysis speed of multi-machine system is slow. Forced oscillation theory in references [6] uses order reduction to express the numerical solution or analysis formula of the electromagnetic torque. The method of solving equations shaft torque greatly reduced order system, but this simplified ignore the interaction between mechanical systems and electrical systems.

On the basis of considering the internal transient process of generator, this paper considers the increase of the order of the equation after adding series compensation capacitors, and taking into account the torque attenuation caused by the loss of the active power in the winding resistance. The influence of the series capacitor on the alternating component of transient electromagnetic torque is analyzed quantitatively. Through time domain simulation, the difference of the initial transient electromagnetic torque of the fault circuit presence or absence of capacitance is verified.

\section{Mathematical modeling of fault component network}

\section{Generator mathematical model}

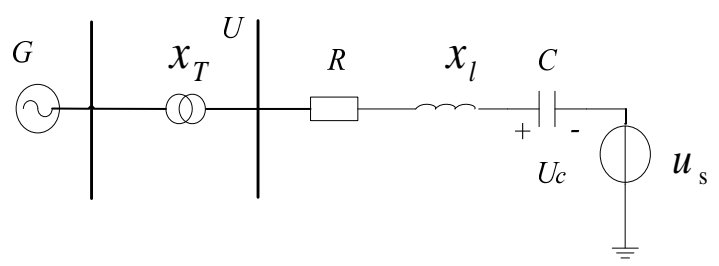

Fig.1 single machine infinite bus system 
Fig. 1 shows the topological structure of a single machine infinite bus system with a series capacitor, the voltage equation set and flux linkage equation set of the d-q axis of the generator are shown in the form of Laplace in formula (1) and (2), the zero-axis component can be ignored.

$$
\begin{aligned}
& \left\{\begin{array}{l}
U_{d}(p)=-r I_{d}(p)+\left(p \Psi_{d}(p)-\psi_{d 0}\right)-\omega \Psi_{q}(p) \\
U_{q}(p)=-r I_{q}(p)+\left(p \Psi_{q}(p)-\psi_{q 0}\right)+\omega \Psi_{d}(p)
\end{array}\right. \\
& \left\{\begin{array}{l}
U_{f}(p)=r_{f} I_{f}(p)+\left(p \Psi_{f}(p)-\psi_{f 0}\right) \\
0=r_{D} I_{D}(p)+\left(p \Psi_{D}(p)-\psi_{D 0}\right) \\
0=r_{Q} I_{Q}(p)+\left(p \Psi_{Q}(p)-\psi_{Q 0}\right)
\end{array}\right. \\
& \left\{\begin{array}{l}
\Psi_{d}(p)=-L_{d} I_{d}(p)+L_{a d} I_{f}(p)+L_{a d} I_{D}(p) \\
\Psi_{q}(p)=-L_{q} I_{q}(p)+L_{a q} I_{Q}(p) \\
\Psi_{f}(p)=-L_{a d} I_{d}(p)+L_{f} I_{f}(p)+L_{a d} I_{D}(p) \\
\Psi_{D}(p)=-L_{a d} I_{d}(p)+L_{a d} I_{f}(p)+L_{D} I_{D}(p) \\
\Psi_{Q}(p)=-L_{a q} I_{q}(p)+L_{Q} I_{Q}(p)
\end{array}\right.
\end{aligned}
$$

In the formula, $\mathrm{r}$ is the resistance, $I_{d}$ and $I_{q}$ are d-axis and q-axis component in stator winding, $I_{f}$ is the exciting current, $I_{D} 、 I_{Q}$ are currents in D and Q damping windings, $\Psi_{f}$ is the excitation winding flux linkage, $\Psi_{D}$ and $\Psi_{Q}$ are flux linkages in D and Q damping winding, $\omega$ is the actual speed of rotor introduced by Park transformation. $L_{d} 、 L_{q}$ are synchronous inductances of d-axis and q-axis, $L_{a d} 、 L_{a q}$ are mutual inductances between rotor windings and stator windings. Through the simultaneous solution of the two sets of equations, using the Gauss elimination method to eliminate the rotor component, the flux and voltage equations which only contain stator winding variables are obtained.

Network mathematical model

Listing voltage equations set of the network and change these equations from abc coordinate system to dq0 coordinate system, as shown in formula (3). Eliminating the voltage of capacitor $U_{C d}$ and $U_{C q}$, as shown in formula (4).

$$
\begin{aligned}
& \left\{\begin{array}{l}
R I_{d}(p)+L\left(p I_{d}(p)-\omega I_{q}(p)\right)+U_{C d}(p)=U_{d}(p)-U_{\infty d}(p) \\
R I_{q}(p)+L\left(p I_{q}(p)+\omega I_{d}(p)\right)+U_{C q}(p)=U_{q}(p)-U_{\infty q}(p) \\
C\left(p U_{C d}(p)-\omega U_{C q}(p)\right)=I_{d}(p) \\
C\left(p U_{C q}(p)+\omega U_{C d}(p)\right)=I_{q}(p)
\end{array}\right. \\
& \left\{\begin{array}{l}
U_{d}(p)=R I_{d}(p)+L\left(p I_{d}(p)-\omega I_{q}(p)\right)+\frac{p I_{d}(p)+\omega I_{q}(p)}{\left(p^{2}+\omega^{2}\right) C}+U_{o d}(p) \\
U_{q}(p)=R I_{q}(p)+L\left(p I_{q}(p)+\omega I_{d}(p)\right)+\frac{p I_{q}(p)-\omega I_{d}(p)}{\left(p^{2}+\omega^{2}\right) C}+U_{o q}(p)
\end{array}\right.
\end{aligned}
$$

In the formula, $I_{d}$ and $I_{q}$ are line currents after transformed to d-axis and q-axis, $\mathrm{L}$ is the line equivalent inductance, $\mathrm{C}$ is the line series compensation capacitor.

Fault network model of three-phase short circuit fault

The superposition theorem can be used as the superposition of normal network and fault component network after three phase short circuit fault in the network. The fault component network 
is shown in Fig.2, where the fault point voltage suddenly drops to zero, which is equivalent to a sudden applied voltage source $-u_{|0|}$ and generator induction electromotive force is 0 at the short circuit point.

Assuming that the excitation voltage and the speed of machine are approximately constant, the generator has no excitation source and the rotor speed of the rotor is kept in the fault component network.

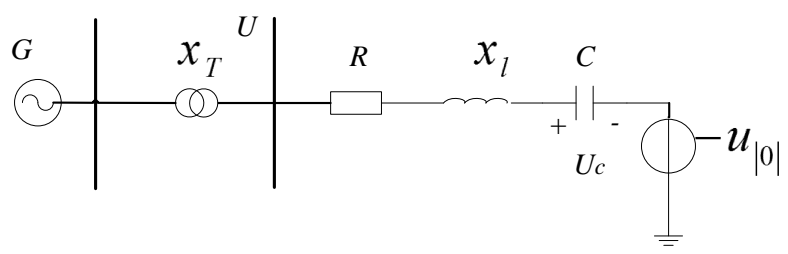

Fig.2 fault component network of single-machine infinite-bus system

When the pre-fault speed is synchronous, reactance and inductance per unit values are the same, reactance can be widely used to express the inductance of the generator and the network, and the network equations of the fault component are shown in formula (5).

$$
\begin{aligned}
-\frac{u_{d|0|}}{p} & =-\left[(r+R)+p\left(X_{d}(\mathrm{p})+X+\frac{1}{\left(\mathrm{p}^{2}+1\right) \mathrm{C}}\right)\right] \Delta \mathrm{I}_{d}(\mathrm{p})+\left(X_{q}(\mathrm{p})+X-\frac{1}{\left(\mathrm{p}^{2}+1\right) \mathrm{C}}\right) \Delta \mathrm{I}_{q}(\mathrm{p}) \\
-\frac{u_{q|0|}}{p} & =-\left[(r+R)+p\left(X_{q}(\mathrm{p})+X+\frac{1}{\left(\mathrm{p}^{2}+1\right) \mathrm{C}}\right)\right] \Delta \mathrm{I}_{q}(\mathrm{p})-\left(X_{d}(\mathrm{p})+X-\frac{1}{\left(\mathrm{p}^{2}+1\right) \mathrm{C}}\right) \Delta \mathrm{I}_{d}(\mathrm{p})
\end{aligned}
$$

\section{Initial value of transient torque}

Solving equations (5), the Laplace representation of the fault component current is obtained, and the corresponding original function is obtained by Laplace inverse transformation, as is shown in formula (6).

$$
\begin{aligned}
& \Delta i_{d}=\frac{u_{|0|} \cos (\delta)}{\left(x^{\prime \prime}+X\right)(1-k)}-\frac{u_{|0|}}{2\left(x^{\prime \prime}+X\right)}\left(\frac{\cos \left(\omega_{d} t+\delta\right)}{\omega_{d}}+\frac{\cos \left(\omega_{g} t+\delta\right)}{\omega_{g}}\right) \\
& \Delta i_{q}=-\frac{u_{|0|} \sin (\delta)}{\left(x^{\prime \prime}+X\right)(1-k)}+\frac{u_{|0|}}{2\left(x^{\prime \prime}+X\right)}\left(\frac{\sin \left(\omega_{d} t+\delta\right)}{\omega_{d}}+\frac{\sin \left(\omega_{g} t+\delta\right)}{\omega_{g}}\right)
\end{aligned}
$$

In this formula, $\omega_{d}=1-\sqrt{k}$ and $\omega_{g}=1+\sqrt{k}$, represent secondary frequency and overclocking in the fault current respectively, $\delta$ is the angle between pre-fault voltage phasor $\dot{U}_{|0|}$ and no-load electromotive $\dot{E}_{q|0|}$.

The electromagnetic torque of synchronous generator $T_{e}=-i_{d} \psi_{q}+i_{q} \psi_{d}$ will also be changed when the fault occurs, changes in the amount of electromagnetic torque at fault can be seen in formula (7).

$$
\begin{aligned}
& \Delta T_{e}=\left[-\left(\Psi_{q|0|}+\Delta \Psi_{q}\right) \quad\left(\Psi_{d|0|}+\Delta \Psi_{q}\right)\right]\left[\begin{array}{c}
i_{d|0|}+\Delta i_{d} \\
i_{q|0|}+\Delta i_{q}
\end{array}\right]-\left[-\Psi_{q|0|} \quad \Psi_{d|0|}\left[\begin{array}{c}
i_{d|0|} \\
i_{q|0|}
\end{array}\right]\right. \\
& =\left[\begin{array}{ll}
-\Psi_{q|0|} & \Psi_{d|0|}
\end{array}\right]\left[\begin{array}{l}
\Delta i_{d} \\
\Delta i_{q}
\end{array}\right]+\left[\begin{array}{ll}
-\Delta \Psi_{q} & \Delta \Psi_{d}
\end{array}\right]\left[\begin{array}{c}
i_{d|0|} \\
i_{q|0|}
\end{array}\right]+\left[\begin{array}{ll}
-\Delta \Psi_{q} & \Delta \Psi_{d}
\end{array}\right]\left[\begin{array}{c}
\Delta i_{d} \\
\Delta i_{q}
\end{array}\right]
\end{aligned}
$$


Most of the steam turbines are hidden pole machines, the d-axis and the q-axis reactance parameters are approximately the same, so the third variation of the electromagnetic torque can be seen as zero. Further simplification of the expression is shown in formula (8).

$$
\begin{aligned}
& \Delta T_{e} \approx E_{d \phi}{ }^{\prime \prime} \Delta_{d}+E_{q d}{ }^{\prime \prime} \Delta_{q} \\
& =\frac{E_{q}^{\prime \prime} u_{q}}{\left(x^{\prime \prime}+X\right)(1-k)}\left(-\sin \left(\delta-\delta^{\prime \prime}\right)+\frac{1}{2}\left(\omega_{g} \sin \left(\omega_{d} t+\delta-\delta^{\prime \prime}\right)+\omega_{d} \sin \left(\omega_{g} t+\delta-\delta^{\prime \prime}\right)\right)\right) \\
& =-T_{\mid q}+\frac{E_{d q}^{\prime \prime} u_{d q}}{2\left(x^{\prime \prime}+X\right)(1-k)}\left(\omega_{g} \sin \left(\omega_{d} t+\delta-\delta^{\prime \prime}\right)+\omega_{d} \sin \left(\omega_{g} t+\delta-\delta^{\prime \prime}\right)\right) \\
& =-T_{\mid q}+\frac{T_{\mid q}}{2 \sin \left(\delta-\delta^{\prime \prime}\right)}\left(\omega_{g} \sin \left(\omega_{d} t+\delta-\delta^{\prime \prime}\right)+\omega_{d} \sin \left(\omega_{g} t+\delta-\delta^{\prime \prime}\right)\right)
\end{aligned}
$$

Formula (8) shows that, when compensation $k$ is zero, that is, when a short circuit does not contain capacitors, electromagnetic torque variation will consist of the unidirectional torque component and alternating component. When the compensation $k$ is not zero, electromagnetic torque variation will consist of the unidirectional torque component, sub synchronous frequency components and ultra-synchronous frequency component. Unidirectional component is the electromagnetic torque before short-circuited happen, alternating component amplitude is related to sub-transient potential and t sub-transient reactance, and sub-transient potential is related to flux linkage of field winding and damping winding, the mutation will not occur before and after the disturbance. The amplitude of the synchronous component and the ultra-synchronous component in alternating component are inversely proportional to its frequency. If the compensation degree $k$ is larger and closer to 1 , the amplitude of the sub synchronous frequency components of the synchronous frequency is higher, the amplitude of the frequency components of the ultra-synchronous frequency is smaller, the sum of amplitude of the two components is certain.

\section{Transient expression in of electromagnetic torque and its time domain simulation}

From the above analysis, in the form of electromagnetic torque can be simplified to formula (9).

$$
\begin{aligned}
\Delta T_{e} \approx E_{d|0|}{ }^{\prime \prime} & \Delta i_{d}+E_{q|0|}{ }^{\prime \prime} \Delta i_{q} \\
& =-T_{|0|}\left(\left(\frac{x+X}{x^{\prime \prime}+X}-\frac{x+X}{x^{\prime}+X}\right) e^{\frac{t}{T_{d}^{\prime \prime}}}+\left(\frac{x+X}{x^{\prime}+X}-1\right) e^{\frac{t}{T_{d}^{\prime}}}+1\right) \\
& +\frac{E_{|0|}^{\prime \prime} u_{0 \mid}}{2\left(x^{\prime \prime}+X\right)(1-k)}\left(\omega_{g} \sin \left(\omega_{d} t+\delta-\delta^{\prime \prime}\right)+\omega_{d} \sin \left(\omega_{g} t+\delta-\delta^{\prime \prime}\right)\right) e^{-\frac{t}{T_{a}}}
\end{aligned}
$$

Decay time constant usually has the following relationship in formula (10).

$$
\begin{gathered}
T_{d}{ }^{\prime \prime}<T_{d}{ }^{\prime} \\
T_{a}<T_{d}{ }^{\prime}
\end{gathered}
$$

The AC component of the electromagnetic torque decay faster than the DC component, eventually decay to zero when the mechanical angular speed variation is not considered. In fact, the change of the electromagnetic torque will cause the torsional vibration response of the steam turbine shaft, mechanical angular velocity is bound to produce oscillation. Therefore electromagnetic torque expression (9) applies only to analyze the situation since the initial moment of inertia turbine generator shaft and not yet had time to produce a greater change. Using IEEE first standard system model (as shown in Fig.3), the PSCAD time domain simulation is used to verify the transient electromagnetic torque derived from the upper section. In order to compare the transient electromagnetic torque with capacitor or without capacitor in the short circuit, the fault point is set to A and B. Fault type is set to three-phase short circuit. 


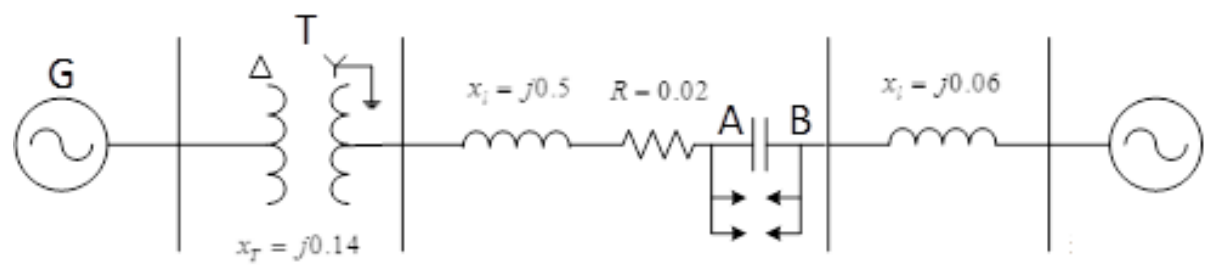

Fig.3 IEEE first standard model wiring diagram

When the fault location is point A and the fault circuit does not contain capacitors, the waveform of the electromagnetic torque is shown in Fig.4. It can be seen that the transient electromagnetic torque contains the $\mathrm{AC}$ component of the power frequency and unidirectional torque component. The initial amplitude of the calculated value is in agreement with the simulation results, the error is only -2.7\%.Table 1 shows the comparison of the calculation and Simulation of the transient electromagnetic torque of the A point at the initial stage when three-phase short circuit happen.

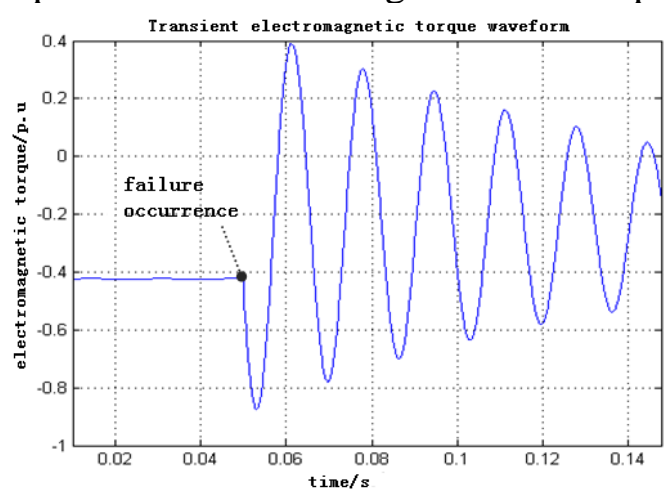

Fig.4 fault transient electromagnetic torque waveform of point $A$

Tab.1 comparison of calculated values and simulation values of point $A$

Simulation value

Calculated value

$\begin{array}{cccc}\text { Frequency(Hz) } & \text { Initial amplitude(p.u) } & \text { Frequency(Hz) } & \text { Initial amplitude(p.u) } \\ 0 & 1 & 0 & 1 \\ 60 & 2.22 & 60 & 2.16\end{array}$

When the fault location is point B and the fault circuit contains capacitors, the waveform of the electromagnetic torque also can be got. It can be seen that the transient electromagnetic torque contains unidirectional torque component, frequency components and ultra-synchronous frequency component. The initial amplitude of the calculated value is in agreement with the simulation results, the error is only $-3.3 \%$ and $-2.5 \%$. Table 2 shows the comparison of the calculation and Simulation of the transient electromagnetic torque of the B point at the initial stage when three-phase short circuit happen.

Tab. 2 comparison of calculated values and simulation values

\begin{tabular}{cccc}
\hline \multicolumn{2}{c}{ Simulation value } & \multicolumn{2}{c}{ Calculated value } \\
\hline Frequency(Hz) & Initial amplitude(p.u) & Frequency(Hz) & Initial amplitude(p.u) \\
0 & 1 & 0 & 1 \\
20 & 2.73 & 18.43 & 2.64 \\
100 & 0.472 & 101.5 & 0.46 \\
\hline
\end{tabular}




\section{Conclusions}

In this paper, the initial form of three-phase short-circuit transient electromagnetic torque is derived under certain assumptions, and the simulation is verified by time domain simulation. The transient electromagnetic torque of the generator is composed of unidirectional torque component and alternating component after three phase short circuit. When fault circuits contain capacitors, alternating component will consist of sub synchronous frequency components and ultra-synchronous frequency component. The ratio of the amplitude between the sub synchronous component and the ultra-synchronous component is inversely proportional to the ratio of their frequency. When the short circuit does not include capacitors, the alternating component is equal to the power frequency component. Based on the results of this paper, the electromagnetic torque equation can be brought into the system of decoupled modal axis system, and the disturbance of the system can be obtained.

\section{References}

[1] IEEE Subsynchronous Resonance Working Group. Proposed terms and definitions for subsynchronous oscillations [J]. IEEE Trans. on Power Appar. Syst, 1980, 99: 506-511.

[2] Chen Deiwei, Li Xingyuan. Synchronous damping controller design based on the matrix pencil algorithm [J]. Proceedings of the CSU-EPSA, 2013, 25(2):37-41. (In Chinese)

[3] Shi Libao,Dai Shiqiang,Xu Zheng, et al. System transient stability simulation of large scale doubly fed wind farm[J]. Proceedings of the CSU-EPSA, 2009, 21(4): 1-7. (In Chinese)

[4] John S.Joyce, Tadeusz Kulig, Dietrich Lambrecht. Torsional fatigue of turbine-generator shafts caused by different eletrical system faults and switching operations [J]. IEEE Transaction on Power Apparatus and Systems, 1978, 97(5): 1965-1977.

[5] Gao Xun, Xie Xiaorong, Wu Tao, et al. Improvement and application of next time synchronous resonance simulation model under large disturbance [J]. Power System Technology, 2012, 36(1): 96-101. (In Chinese)

[6] A.Abolins, D.Lambrecht, J. S. Joyce, et al. Effect of clearing short circuits and automatic reclosing on torsional stress and life expenditure of turbine-generator shafts [J]. IEEE Transaction on Power Apparatus and Systems, 1976, 95 (1) : 14-25. 